\title{
Contribution of rpfB to Cell-to-Cell Signal Synthesis, Virulence, and Vector Transmission of Xylella fastidiosa
}

\author{
Rodrigo P. P. Almeida, ${ }^{1}$ Nabil Killiny, ${ }^{2}$ Karyn L. Newman, ${ }^{3}$ Subhadeep Chatterjee, ${ }^{4}$ Michael lonescu, ${ }^{3}$ \\ and Steven E. Lindow ${ }^{3}$
}

\begin{abstract}
${ }^{1}$ Department of Environmental Science, Policy, and Management, University of California, Berkeley 94720, U.S.A.; ${ }^{2}$ Department of Entomology and Nematology, Citrus Research and Education Center, University of Florida, Lake Alfred 33850, U.S.A.; ${ }^{3}$ Department of Plant and Microbial Biology, University of California, Berkeley; ${ }^{4}$ Centre for DNA Fingerprinting and Diagnostics, Hyderabad 500001, India
\end{abstract}

Submitted 24 March 2011. Accepted 9 December 2011.

In Xylella fastidiosa the fatty acid signal molecule diffusible signaling factor (DSF) is produced and sensed by components of the regulation of pathogenicity factors (rpf) cluster; lack of DSF production in RpfF mutants results in a nonvector-transmissible phenotype yet cells are hypervirulent to grape. $r p f B$ has not been characterized in Xylella fastidiosa, although its homolog has been suggested to be required for DSF synthesis in Xanthomonas campestris pv. campestris. We show that RpfB is involved in DSF processing in both Xylella fastidiosa and Xanthomonas campestris, affecting the profile of DSF-like fatty acids observed in thin-layer chromatography. Although three fatty acids whose production is dependent on RpfF were detected in Xylella fastidiosa and Xanthomonas campestris wild-type strains, their respective $r p f B$ mutants accumulated primarily one chemical species. Although no quantifiable effect of rpfB on plant colonization by Xylella fastidiosa was found, insect colonization and transmission was reduced. Thus, RpfB apparently is involved in DSF processing, and like Xanthomonas campestris, Xylella fastidiosa also produces multiple DSF molecules. It is possible that Xylella fastidiosa coordinates host vector and plant colonization by varying the proportions of different forms of DSF signals via RpfB.

Cell-to-cell signaling and its role in the biology of a wide variety of bacteria, especially pathogenic organisms, have been well documented. The concentration of signaling molecules serves as a proxy for population size in a diffusion-limited environment, and triggers changes in gene transcription profiles, such as the expression of virulence factors in human and plant pathogens (Camilli and Bassler 2006). Such coordination of transcription has been shown to be essential in pathogens, for example, for successful colonization of hosts, biofilm formation, and vector transmission (Dow et al. 2003; Newman et al. 2004). Importantly, cell-to-cell signaling is important for the success of pathogens with complex life histories and multiple hosts, such as Vibrio cholerae, which, in addition to human hosts, also colonizes the surface of arthropods in aquatic environments (Pruzzo et al. 2008). Another such microbe is the plant-pathogenic bacterium Xylella fastidiosa, which must colonize the foregut of leafhopper vectors to be spread from plant to plant (Almeida et al. 2005; Chatterjee et al. 2008a). Interestingly, Xylella fastidiosa's cell-to-cell signaling system sup-

Corresponding author: R. Almeida; E-mail: rodrigoalmeida@berkeley.edu; Telephone: +1.510 .642 .1603 ; Fax: +1.510 .643 .5438 . presses virulence in plants, because mutants deficient in signal production are hypervirulent but are deficient in vector colonization and transmission (Newman et al. 2004).

Unlike the better-understood bacterial cell-to-cell signaling systems based on acyl-homoserine lactones, the signal molecule in Xylella fastidiosa, referred to as a diffusible signaling factor (DSF), is apparently one or more fatty acids based on the similarity of its signal production and response genes to that in many Xanthomonas spp. In Xanthomonas campestris pv. campestris (hereafter Xanthomonas campestris), expression of pathogenicity factors is under DSF regulation and signaling-deficient mutants have reduced virulence (Barber et al. 1997). Although DSF from Xanthomonas campestris was initially described as only cis-11-methyl-2-dodecenoic acid, this species is now recognized to also produce $c i s$-dodecenoic acid (BDSF) and a novel unsaturated fatty acid, that is otherwise identical to DSF except for an additional double bond between $\mathrm{C}_{5}$ and $\mathrm{C}_{6}$ (CDSF) (He et al. 2010). The identity of the DSF molecule made by Xylella fastidiosa, while currently unknown, is thought to be similar but probably different from those made by Xanthomonas spp., because a Xanthomonas campestris DSF biosensor strain recognizes DSF produced by Xylella fastidiosa, although weakly (Chatterjee et al. 2008b; Newman et al. 2004). In both pathogens, the several genes associated with DSF production and sensing (regulation of pathogenicity factors [rpf]) have high homology and are organized in clusters although, in $X y l e l l a$ fastidiosa, two genes ( $r p f A$ and $r p f B$ ) are located elsewhere in the chromosome (Fig. 1). rpfA encodes an aconitase and has been implicated in the regulation of gene expression in Xanthomonas campestris (Wilson et al. 1998). Xanthomonas campestris $\mathrm{RpfB}$ is predicted to be a long-chain fatty acyl CoA ligase, and has been suggested to be essential for the synthesis of DSF, in conjunction with the enoyl CoA hydratase RpfF (Barber et al. 1997; Cheng et al. 2010). Other components of the signaling pathway in Xanthomonas campestris have been characterized (Deng et al. 2011; Dow 2008; He and Zhang 2008) but only RpfF and RpfC have been studied in detail in Xylella fastidiosa. The role of these proteins in Xylella fastidiosa is apparently similar to that in Xanthomonas campestris, with RpfF required for synthesis of DSF (Newman et al. 2004) and RpfC being a sensor for DSF that is part of a putative two-component regulatory scheme (Chatterjee et al. 2008b). The DSF-mediated regulation of virulence factors seems to be largely responsible for the control of pathogenicity factors associated with host plant colonization in Xanthomonas campestris, with RpfF and RpfB associated with DSF production (Barber et al. 1997) and RpfC and RpfG with signal perception 
and transduction (Dow 2008; He and Zhang 2008; He et al. 2006), leading to downstream control of gene transcription via modulation of cyclic di-GMP levels.

In addition to controlling Xylella fastidiosa colonization of plants, DSF also regulates this pathogen's ability to colonize its insect vectors, an essential component of its biology (Newman et al. 2004). Cells must attach to and colonize the cuticular surface of specific regions of the vector's foregut, forming microcolonies that eventually develop into mature biofilms of polarly attached cells (Almeida and Purcell 2006). A DSFdeficient RpfF mutant was not able to colonize the foregut of vectors and was not transmissible to plants (Newman et al. 2004). On the other hand, an $r p f C$ mutant, which overproduces DSF, was hyperadhesive in vitro compared with the wild type, and was transmissible by vectors with low efficiency, possibly because it was not detachable from vectors as easily as the wild type (Chatterjee et al. 2008b). Mutants blocked in expression of a cyclic di-GMP synthase ( $c_{s} A$, which has a GGDEF domain), putatively involved in cyclic di-GMP synthesis that was downregulated in the presence of DSF, also exhibited a hyper adhesive phenotype, and were only weakly virulent to plants while transmissible by insect vectors (Chatterjee et al.
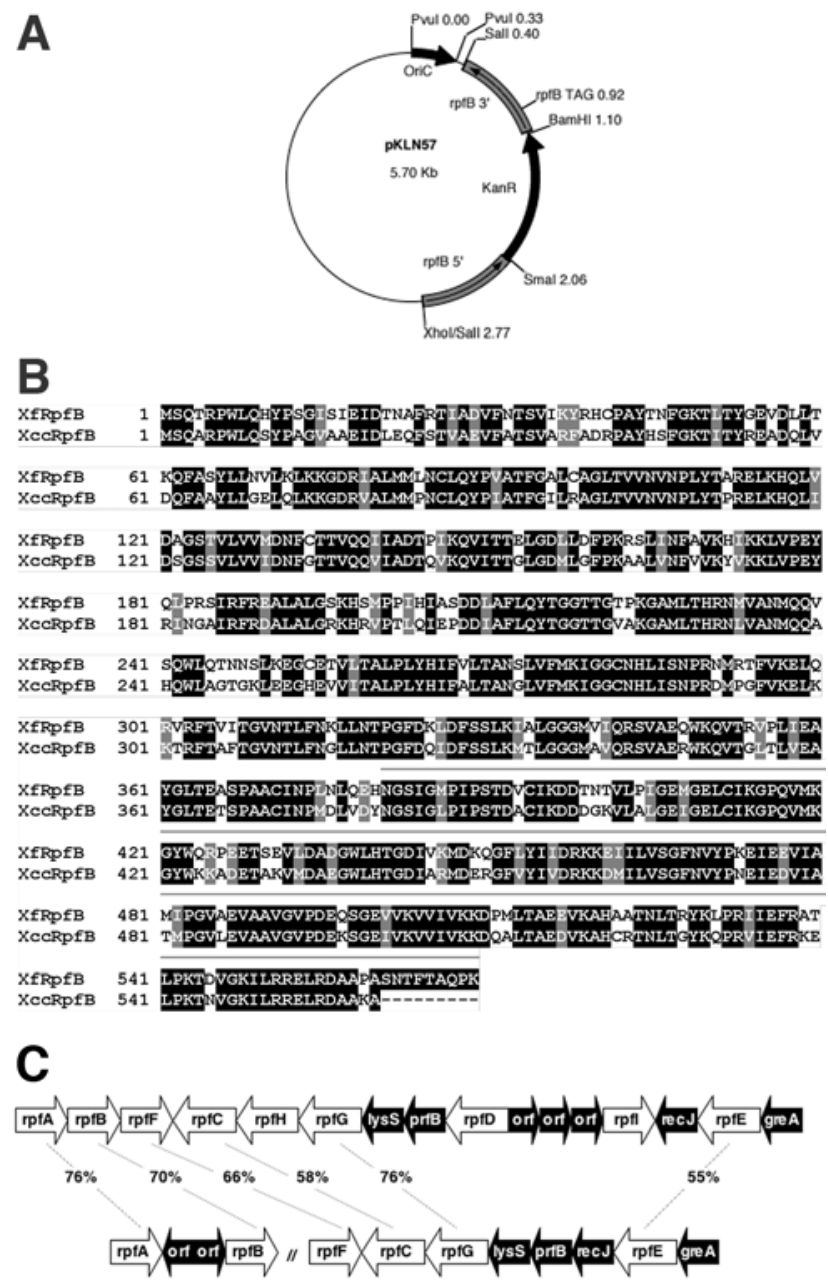

Fig. 1. A, Structure of plasmid $p$ KLN57 used to create $r p f B$ mutant. Gray shaded areas represent $5^{\prime}$ and $3^{\prime}$ regions of $r p f B$ gene, which had a coding sequence removed and replaced with the kanamycin resistance $\left(\operatorname{Kan}^{\mathrm{r}}\right)$ gene. B, Alignment of the predicted amino acid sequences of $r p f B$ from Xylella fastidiosa and Xanthomonas campestris showing amino acid identity (dark regions) or similarity (gray regions). C, Genomic organization of the regions harboring rpf genes in Xanthomonas campestris (top) and that of Xylella fastidiosa (bottom). Note that $r p f A$ and $r p f B$ in Xylella fastidiosa are unlinked to the rests of the rpf genes, unlike in Xanthomonas campestris.
2010). These results suggest that decreases of cyclic di-GMP result from the accumulation of DSF as cell density increases, leading to a phenotypic transition from a planktonic state capable of colonizing host plants to an adhesive state that is insect transmissible. Afimbrial adhesins such as hemagglutinin-like proteins are upregulated by DSF and are important for initial cell attachment to vectors (Killiny and Almeida 2009a and b), a process initiated when colonies in plants reach high densities, limiting migration of Xylella fastidiosa within the xylem network of hosts but facilitating their adhesion to insects (Guilhabert and Kirkpatrick 2005, Killiny and Almeida 2009b). On the other hand, genes that would facilitate movement of the pathogen and growth in plants such as those conferring production of long pili and extracellular enzymes are upregulated in the absence of DSF (Chatterjee et al. 2008b) and do not seem to affect vector transmission (Killiny and Almeida 2009b), suggesting that cell-to-cell signaling controls the dual lifestyle of this bacterium (Chatterjee et al. 2008a). The traits that would contribute to insect colonization by Xylella fastidiosa seem to conflict with plant colonization; therefore, spatially structured populations of Xylella fastidiosa may occur within xylem vessels, and the dichotomous characteristics of these phenotypical states may be regulated by differential accumulation of DSF (Chatterjee et al. 2008a). Because there is no known insect vector of Xanthomonas pathogens, gene regulation, while involving DSF, may not be as complex as that in Xylella fastidiosa.

We speculate that the distal location of $r p f B$ from the $r p f$ operon in Xylella fastidiosa may reflect a need for more complex regulation of the rpf-regulated traits from that in Xanthomonas campestris. Little is known of the role of RpfB in DSFmediated cell signaling except for an initial report indicating that it is required for DSF synthesis in Xanthomonas campestris (Barber et al. 1997). Given that $r p f B$ was itself responsive to the presence of DSF (Chatterjee et al. 2008a), we speculated that its role may be more complex than originally thought. In this study, we characterized the biological role of $r p f B$ in $X y$ lella fastidiosa. We tested the hypothesis that it would have a role in DSF synthesis as essential as its homologue in Xanthomonas campestris. We found, however, that $\operatorname{rpfB}$ did not affect overall DSF activity in either Xylella fastidiosa or Xanthomonas campestris, and that it apparently modulates the composition of a mixed family of related signal molecules that are produced. In addition, while only modest differences in plant colonization were seen between the wild type and an $r p f B$ mutant strain when mechanically inoculated, vector colonization and transmission were impaired. These results suggest that RpfB plays a role in modulating the chemical composition of cellular DSF species that, in turn, may influence patterns of gene regulation and, thus, the behavior of Xylella fastidiosa.

\section{RESULTS}

DSF synthesis in Xylella fastidiosa does not require RpfB.

A Xylella fastidiosa rpfB mutant was made by allelic exchange using pKLN57 (Fig. 1A). Xylella fastidiosa rpfB is highly similar to long-chain fatty acid acyl coA ligases, which play roles in lipid metabolism and secondary metabolite biosynthesis, transport, and catabolism. The $r p f B$ mutant strain KLN57 lacks $r p f B$ codons 321 to 569 ; this deleted region is the most highly conserved region in RpfB, suggesting that it is essential for protein function. Because no genes are predicted to be transcribed immediately downstream of $r p f B$, it is likely that strain KLN57 harboring an insertion in $r p f B$ does not confer a polar effect on adjacent genes. DSF reporter strain 8523 (Xanthomonas campestris) exhibited green fluorescent protein (GFP) fluorescence when exposed to ethyl acetate extracts of 
KLN57 culture supernatants similar to that of extracts of wildtype Xylella fastidiosa, indicating that KLN57 was unimpaired in DSF synthesis (Fig. 2). Extracts of cell-free media and from the $r p f F$ mutant (KLN61) induced no response from the DSF reporter.

\section{Plasmid-expressed $r p f B$ cannot restore DSF production to a Xanthomonas campestris rpfB mutant.}

To test the function of Xylella fastidiosa rpfB, we attempted to rescue a Xanthomonas campestris rpfB mutant using a constitutively expressed $r p f B$ from Xylella fastidiosa in trans. $r p f B$ rescue plasmid pKLN72, which carries $r p f B$ from Xylella fastidiosa under the control of the lac promoter, was introduced into Xanthomonas campestris rpfB mutant strain 8550. As a positive control, Xanthomonas campestris $r p f B$ was introduced on an identical plasmid, pKLN71. Surprisingly, neither of these rescue constructs restored DSF production as detected by induction of an eng:gfp reporter gene in the Xanthomonas campestris rpfB mutant (Fig. 3). In Xanthomonas campestris, $r p f F$ lies downstream of $r p f B$ in the same orientation. Thus, mutations in Xanthomonas campestris disrupting rpfB apparently have a polar effect on the downstream $r p f F$.

\section{$r p f F$ restores DSF synthesis}

to Xanthomonas campestris rpfB mutants.

To test whether adding $r p f F$ gene function could alone restore DSF production in Xanthomonas campestris rpfB mutants, we mated the rpfF rescue plasmids pKLN69 and pKLN70, which carry the Xanthomonas campestris and Xylella fastidiosa $r p f F$ genes, respectively, into strain 8550 . Indeed, both of these plasmids were able to restore production of DSF to the Xanthomonas campestris rpfB mutant (Fig. 3). These results suggest that only $r p f F$ is required and sufficient for DSF production in Xanthomonas campestris as well as Xylella fastidiosa. We also examined the expression of $r p f F$ in the wild type and $r p f B$ background by reverse-transcriptase real-time polymerase chain reaction (PCR) analysis. A relatively small twofold increase in expression of $r p f F$ in the $r p f B$ mutant was observed (Table 1).
Fatty acid production in $r p f B$ and $r p f F$ mutants of Xanthomonas campestris and Xylella fastidiosa.

We explored whether RpfB serves as an acyl coA ligase and, thus, plays a role in providing suitable substrates for RpfF or to alter the products of RpfF, a DSF synthase. We hypothesized that, whereas the aggregate amount of signal molecule production by an $r p f B$ mutant may be similar to that of a wild-type strain of Xanthomonas campestris, the mixtures of fatty acids produced might differ, as resolved by thin-layer chromatography

Table 1. Expression of virulence genes in an $\operatorname{rpfB}$ and $\operatorname{rpf} F$ mutants of Xylella fastidiosa

\begin{tabular}{lcc}
\hline & \multicolumn{2}{c}{ Relative expression $^{\mathbf{a}}$} \\
\cline { 2 - 3 } Gene $^{\mathbf{b}}$ & $\boldsymbol{r p \boldsymbol { f } \boldsymbol { B } \text { mutant }}$ & $\boldsymbol{r p \boldsymbol { f } \boldsymbol { F } \text { mutant } ^ { \mathbf { c } }}$ \\
\hline gumJ & $4.5 \pm 0.5$ & $0.56 \pm 0.02$ \\
fimA & $5.3 \pm 0.1$ & $0.4 \pm 0.04$ \\
hxfA & $0.6 \pm 0.02$ & n.d. \\
hxfB & $1.6 \pm 0.06$ & $0.15 \pm 0.05$ \\
xadA & $2.2 \pm 0.08$ & $0.56 \pm 0.07$ \\
pilY1 & $6.3 \pm 0.7$ & n.d. \\
rpfF & $2.0 \pm 0.02$ & n.d. \\
rpfC & $1.7 \pm 0.01$ & $4.9 \pm 0.4$ \\
chiA & $1.8 \pm 0.02$ & n.d. \\
pglA & $0.7 \pm 0.01$ & $1.9 \pm 0.17$ \\
cgsA & $2.6 \pm 0.02$ & $5.3 \pm 0.3$ \\
tolC & $1.2 \pm 0.01$ & $5.5 \pm 0.7$ \\
engxcA & $0.3 \pm 0.01$ & n.d. \\
\hline
\end{tabular}

${ }^{\text {a }}$ Expression of an $\operatorname{rpf} B$ mutant relative to that of a wild-type strain of $X$. fastidiosa is shown (mean rations of replications \pm standard error; n.d. $=$ not detected). Amount of RNA relative to that in wild-type cells is equal to 1.0 , and was normalized for population size using 16S rRNA as an endogenous control.

${ }^{\mathrm{b}}$ Gene designations (described or putative): gumJ = exopolysaccharide polymerization protein, fimA = type I pili short major subunit, $h x f A$ and $\mathrm{B}=$ hemagglutinin-like proteins $\mathrm{A}$ and $\mathrm{B}, x a d A=$ outer membrane protein, pilY1 = type IV pili tip adhesion protein, $r p f F=$ enoyl CoA hydratase, $r p f C=$ hybrid sensor kinase, $\operatorname{chi} A=$ chitinase $\mathrm{A}, p g l A=$ polygalacturonase, $\operatorname{cgs} A=$ cyclic di-GMP synthase $\mathrm{A}$, tol $C=$ multidrug resistance efflux pump protein, engxcA = endo-1,4- $\beta$-glucanase.

c Data from Chatterjee and associates (2008b).
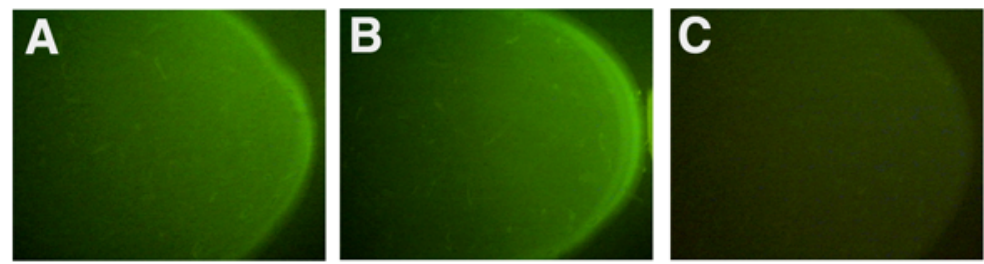

Fig. 2. Production of diffusible signaling factor (DSF) by an rpfB mutant of Xylella fastidiosa detected with an eng:gfp reporter gene in Xanthomonas campestris. DSF presence in culture extracts of $\mathbf{A}$, the wild-type strain of Xylella fastidiosa or $\mathbf{B}$, an rpfB mutant but not from $\mathbf{C}$, uninoculated culture media is apparent as green fluorescence.

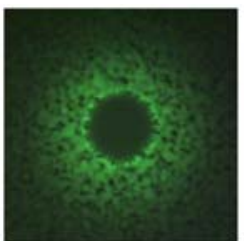

8004

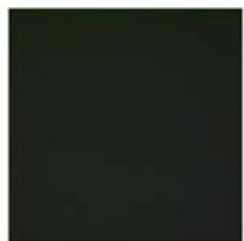

8550

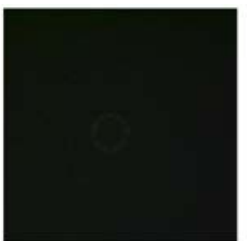

8550 (pKLN71)

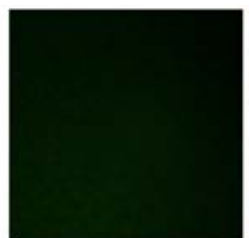

8550 (pKLN72)

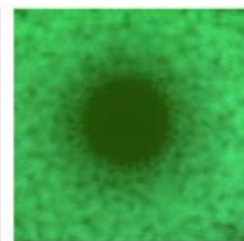

8550 (pKLN69)

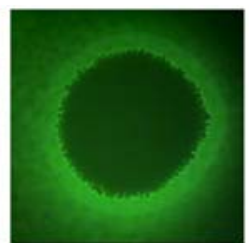

8550 (pKLN70)

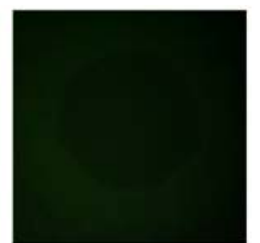

8550

(pBBR1MCS-3)

Fig. 3. Production of diffusible signaling factor (DSF) detected with an eng:gfp reporter gene in Xanthomonas campestris by (from left) colonies of wildtype Xanthomonas campestris 8004, Xanthomonas campestris rpfB mutant 8550, as well as a strain harboring a cloned rpfB gene from Xanthomonas campestris on plasmid pKLN71, a cloned $r p f B$ gene from Xylella fastidiosa on plasmid pKLN72, a cloned rpfF gene from Xanthomonas campestris on plasmid pKLN69, a cloned rpfF gene from Xylella fastidiosa on plasmid pKLN70, and the empty cloning vector pBBR1MCS-3. DSF production is apparent as green fluorescence surrounding a colony. 
(TLC) analysis. Significantly, three molecular species that migrated under these conditions were resolved in extracts of the wild-type strain, while none were found in extracts of an rpfF mutant (Fig. 4). Different proportions of the iodine-reactive material associated with the three different spots were observed in extracts of the $r p f B$ mutant compared with wild-type Xanthomonas campestris; although there was a great reduction in the abundance of the slowest migrating spot and a nearly complete abolishment of the fastest-migrating spot, there was a large increase in the amount of the compound having an intermediate rate of migration in extracts of the $\operatorname{rpf} B$ mutant compared with the wild-type strain (Fig. 4). These results suggest strongly that the relative amounts of the RpfF-dependent fatty acids differed greatly between the wild-type and $r p f B$ mutant strains. The identity of the three different molecular species could not be confirmed but preliminary evidence suggested that the spot with an intermediate rate of migration co-migrated with authentic cisdodecenoic acid (BDSF). We presume that, due to its high relative abundance compared with the other molecular species, the slow migrating spot may conform to cis-11-methyl-2-dodecenoic acid (DSF), because this was the most abundant molecule found in Xanthomonas campestris (He et al. 2010).

RpfB also appears to modulate the relative amounts of RpfF-dependent fatty acids extractable from cultures of $X y$ lella fastidiosa. As in Xanthomonas campestris, three molecular species that migrated under these conditions were resolved in extracts of a wild-type strain of Xylella fastidiosa, while none were found in extracts of an $r p f F$ mutant (Fig. 4). A large difference in the proportion of the iodine-reactive material that was associated with the three different spots was observed in extracts of an $r p f B$ mutant compared with a wild-type Xylella fastidiosa strain; although there was nearly complete absence of the abundant, slowest migrating spot as well as the fastestmigrating spot, there was a large increase in the amount of the spot having an intermediate rate of migration (Fig. 4). Complementation of the $r p f B$ mutant resulted in the recovery of similar amounts of both the fastest-migrant spot and the slowest migrating spot and a substantial reduction of the amount of the intermediate spot to yield a pattern of iodine-reactive compounds that closely resembled that of the wild-type strain (data not shown). The spots from extracts of Xanthomonas campestris and Xylella fastidiosa with an intermediate rate of migration exhibited a similar retardation factor $\left(\mathrm{R}_{\mathrm{f}}\right)$ in this TLC sys-

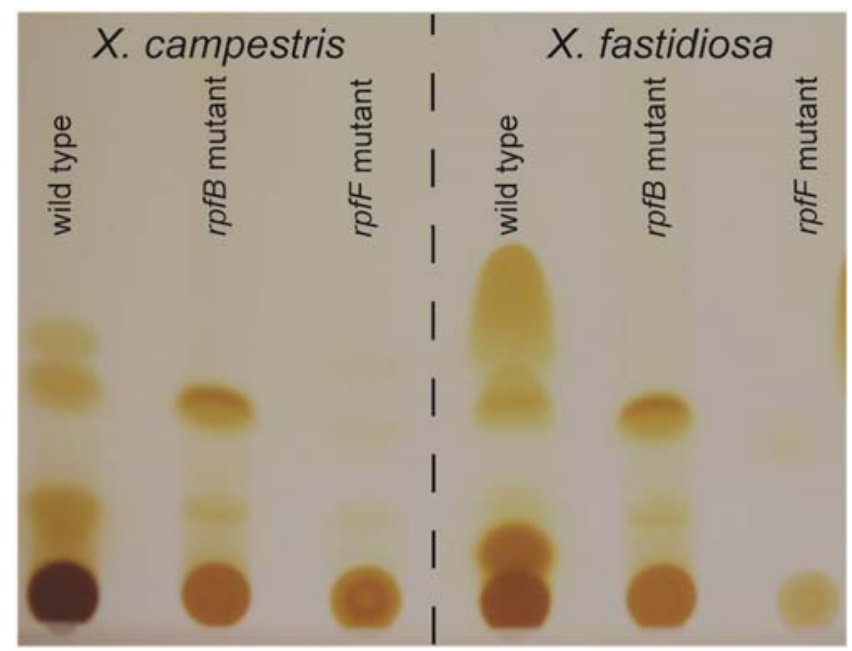

Fig. 4. Thin-layer chromatography of diffusible signaling factor extracted from broth cultures of Xanthomonas campestris (left) and Xylella fastidiosa (right), including their respective wild-type, $r p f B$, and $r p f F$ mutant strains. Samples were spotted at the bottom (dark spots) and fatty acids migrate upward. tem, whereas the fast- and slow-migrating spots differed substantially from each other in their respective $R_{f}$ values. If the spot with intermediate $\mathrm{R}_{\mathrm{f}}$ is, indeed, BDSF, this could account for the fact that little difference in apparent signal molecule production was noted between the wild type and $r p f B$ mutants of these two species because this molecule is nearly as easily detectable as DSF itself using Xanthomonas campestris-based DSF biosensors (Wang et al. 2004).

Because the composition of putative fatty acid signal molecules differed between wild-type and $r p f B$ mutant strains of $X y l e l l a$ fastidiosa, we explored any differences in gene expression that might be associated with this change in relative abundance to test the hypothesis that the various signal molecules could mediate differential patterns of gene expression due to varied interactions with the signal transduction system. The expression of several genes encoding cell-surface adhesins, components of twitching motility, and of extracellular enzymes and compounds were compared in the wild type and an $r p f B$ mutant. Although the expression of many of the genes did not differ substantially, some, such as gumJ, fimA, and pilY1, were highly upregulated in the $r p f B$ mutant compared with the wildtype strain, whereas engxcA was downregulated (Table 1).

\section{Virulence of $r p f B$ mutants.}

We compared the ability of the Xylella fastidiosa $r p f B$ mutant strain KLN57 and the wild-type strain to cause Pierce's disease in grapevine after mechanical inoculation into stems. The number of leaves exhibiting leaf scorch symptoms was similar in both sets of plants; by 16 weeks after inoculation, vines infected with the wild-type strain had $4.14 \pm 0.51$ symptomatic leaves while those infected with the $r p f B$ mutant had $5.67 \pm 1.36$ symptomatic leaves. The disease severity incited by these two strains did not differ significantly. Similar results were observed in replicate virulence tests. Because mechanical inoculation using a needle disrupts plant tissue and delivers many more bacterial cells into the host plant than insect-mediated inoculation, we reasoned that a virulence defect may be masked by such inoculation protocols. Therefore, we evaluated the virulence of the $r p f B$ mutant and wild-type strain in insectinoculated grapevine seedlings. We found that, although all

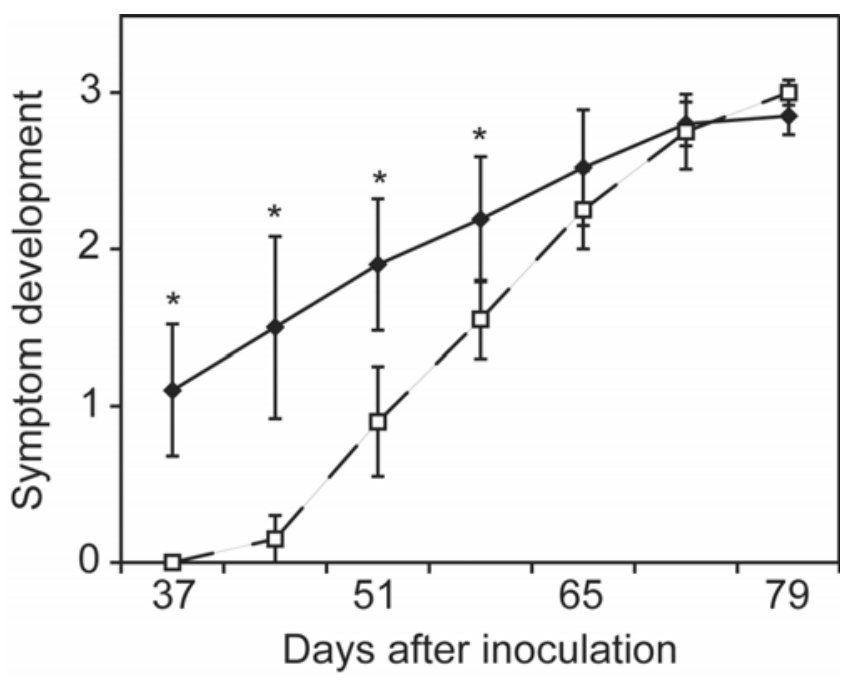

Fig. 5. Pierce's disease symptom development in grapevines vector inoculated with the wild-type (diamonds, solid line) and $r p f B$ mutant (squares, dashed line) strains of Xylella fastidiosa. The leaf scorching disease severity index on plants inoculated as a function of the time after inoculation is shown, and vertical bars represent the standard error of the determination of the mean disease severity. Asterisks indicate statistically significant periods. 
vines exhibited extensive symptoms of Pierce's disease by 11 weeks post inoculation, those infected with the $r p f B$ mutant displayed a lag in symptom development compared with vines infected with Temecula (Fig. 5).

\section{Vector transmission tests.}

To investigate whether $r p f B$ contributes to interactions with insect vectors, we compared the frequency of transmission of the $r p f B$ mutant and the wild-type strain from infected source plants with uninfected target plants. We found that the $r p f B$ mutant and the wild-type strain were transmitted to plants with similar frequencies ( 24 of 46 and 37 of 54 plants, respectively); this difference was not statistically significant $\left(\chi^{2}=2.79, \mathrm{df}=1\right.$, $P=0.095)$. We also evaluated the colonization of leafhoppers by strain KLN57 by both culturing and microscopy. Interestingly, cells were not observed by microscopy in any insects that fed on plants infected with KLN57 but, instead, a network of string-like material was observed in the cibarium and precibarium of many insects (Fig. 6). This material was often quite abundant and might easily have obscured the presence of any cells that might also have been present. In all, $80 \%$ of the individuals that had fed on plants colonized by the wild-type strain had visible bacteria in their mouthparts while $40 \%$ of individuals that had fed on plants infected with KLN57 had the network-like structures only. No cells or other structures were observed in the remaining insects in the negative controls fed on plants not infected with Xylella fastidiosa. These frequencies were not statistically different (Fisher exact test, $P=0.0849$ ) but they were each similar to the frequency at which the insects had transmitted the pathogen to target plants. Although cells of the $r p f B$ mutant were not visible by microscopy of insect heads, they were detectable by culturing of at least some heads. KLN57 was cultured from fewer individuals than the wild-type strain when assayed 1 week after the end of the acquisition period ( 2 of 17 and 11 of 22 insects, respectively; Fisher exact test, $P=0.013$ ). On the other hand, when sampled immediately after leafhopper access to infected plants, the number of vectors carrying the wild-type strain (8 of 19) was larger than for the $\operatorname{rpfB}$ mutant (3 of 19), although not statistically different (Fisher exact test, $P=0.075$ ). It is intriguing that we detected the $r p f B$ mutant strain in only a few of the tested insects ( 5 of 36) and, whereas no cells were observed by microscopy, the frequency of transmission $(56 \%$, discussed above) was higher than the frequency of detection and comparable with that of the wild-type strain.

In a different type of transmission experiment that used sequential transfers of insects from plant to plant, we observed that the wild-type strain was retained by vectors for up to 16 days after acquisition, with no change in transmission rate over time (Fig. 7). On the other hand, transmission rates of the $r p f B$ mutant declined after subsequent sequential transfers to healthy plants, suggesting that colonization of the foregut of vectors is defective and affects transmission. These data corroborate the results presented above, suggesting that, while initial adhesion of to the mouthparts of vectors is not affected in the $r p f B$ mutant, subsequent biofilm maturation and vector colonization are impacted by this mutation.

\section{DISCUSSION}

Our understanding of cell-to-cell signaling in Xanthomonas spp. and Xylella fastidiosa has benefited from a better understanding of the nature of the signal molecules known as DSF. Although some of the observations made in the earliest studies of DSF-mediated cell signaling had suggested that more than one fatty acid may participate in transcriptional regulation of virulence genes in Xanthomonas campestris (Barber et al. 1997), a more recent reexamination of the chemical identify of DSF in Xanthomonas oryzae pv. oryzae and Xanthomonas campestris has revealed that both species produce mixtures of at least three different but closely related fatty acids that participate in regulation of virulence factors (He et al. 2010). Although this study did not address whether each of the chemical
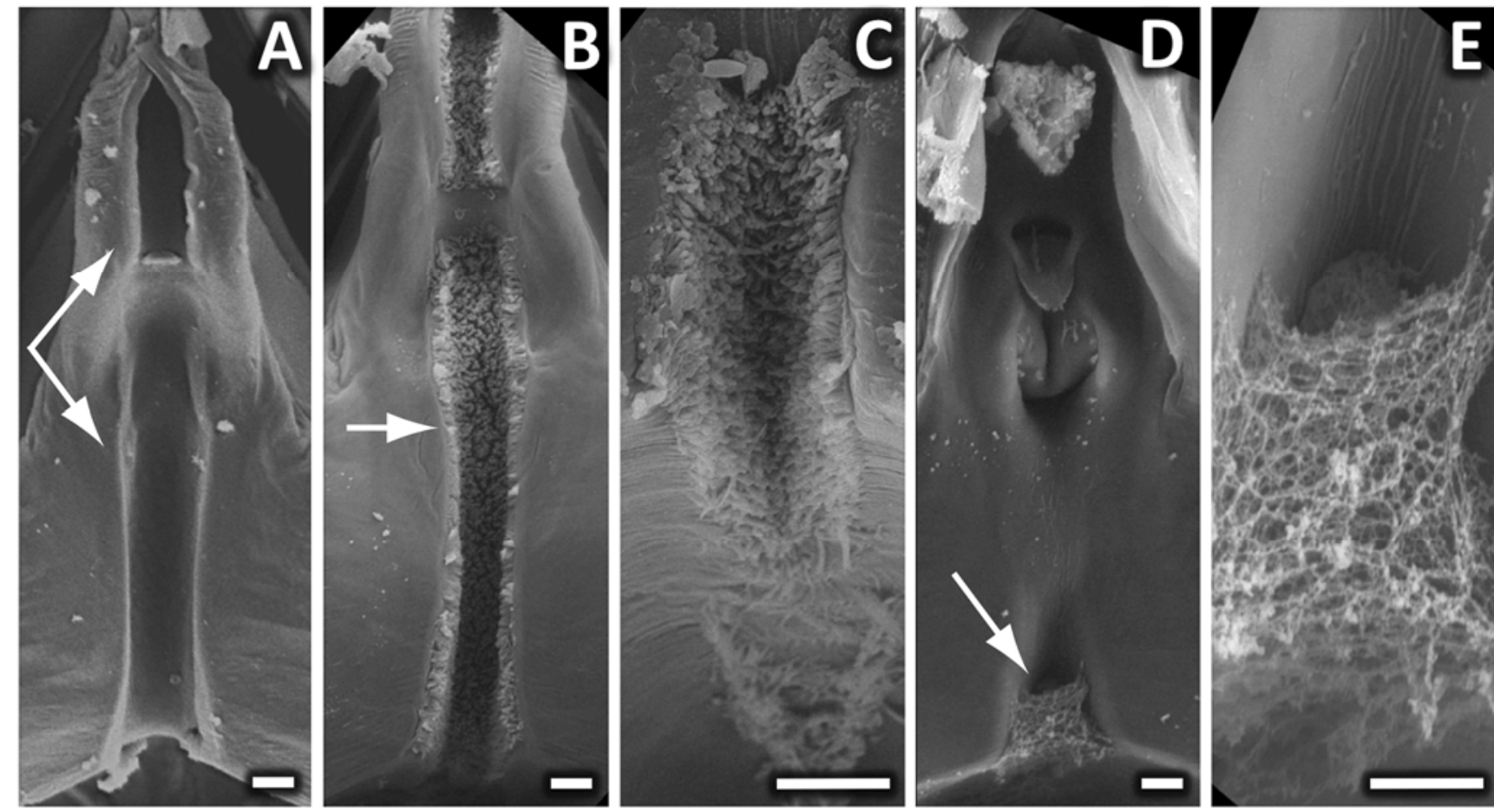

Fig. 6. Colonization of the foregut of leafhopper vectors. A, Uninfected control; $\mathbf{B}$ and $\mathbf{C}$, the foregut of leafhopper vectors fed on plants infected by the Xylella fastidiosa wild-type; or $\mathbf{D}$ and $\mathbf{E}, r p f B$ mutant strains. Colonization of the precibarium (A, B, and D) and proximal (toward cibarium) region of the precibarium ( $\mathrm{C}$ and $\mathrm{E}$ ) of leafhoppers is shown. Precibarium (long canal) is shown with arrows in A, colony of cells lining the precibarium is indicated by an arrow in B, fimbriae-like complex is indicated by an arrow in D, and that same region is expanded in E. Bars $=5 \mu \mathrm{m}$. 
species mediated a similar response in the producing strain, the results shown here suggest that they may not. That is, it seems apparent that considerations of DSF-mediated signaling must acknowledge that more than one chemical species may be involved, and that the different chemical species may preferentially be involved in regulating certain phenotypes in these pathogens. The results of this study show that RpfB, previously thought to be required for synthesis of DSF together with RpfF, is not, in fact, absolutely required for DSF synthesis but, instead, plays a role only in modulating the mixture of chemical species of DSF molecules that are produced in both Xylella fastidiosa and Xanthomonas campestris.

Assays that revealed that the Xylella fastidiosa rpfB mutant did not differ from the wild-type strain in induction of a DSFdependent eng:gfp reporter gene in Xanthomonas campestris may have been somewhat misleading, because the mixture of chemical species produced by the $r p f B$ mutant was apparently quite altered. Induction of the DSF Xanthomonas campestris biosensor by the extracts of the Xylella fastidiosa rpfB mutant indicates that the altered pool of fatty acid produced is still able to induce the Xanthomonas campestris biosensor; however, it may have a different biological effect in virulence gene expression regulation. Expression analysis also indicated that $r p f F$ and $r p f B$ regulate a subset of genes in a differential manner. It also seems clear that the original finding that $\mathrm{RpfB}$ is required for DSF production per se (Barber et al. 1997) was due to the fact the $\operatorname{rpf} B$ mutation resulted in a polar effect on the downstream $r p f F$, thus abolishing its activity. This is supported by the finding that a Xanthomonas campestris DSF-

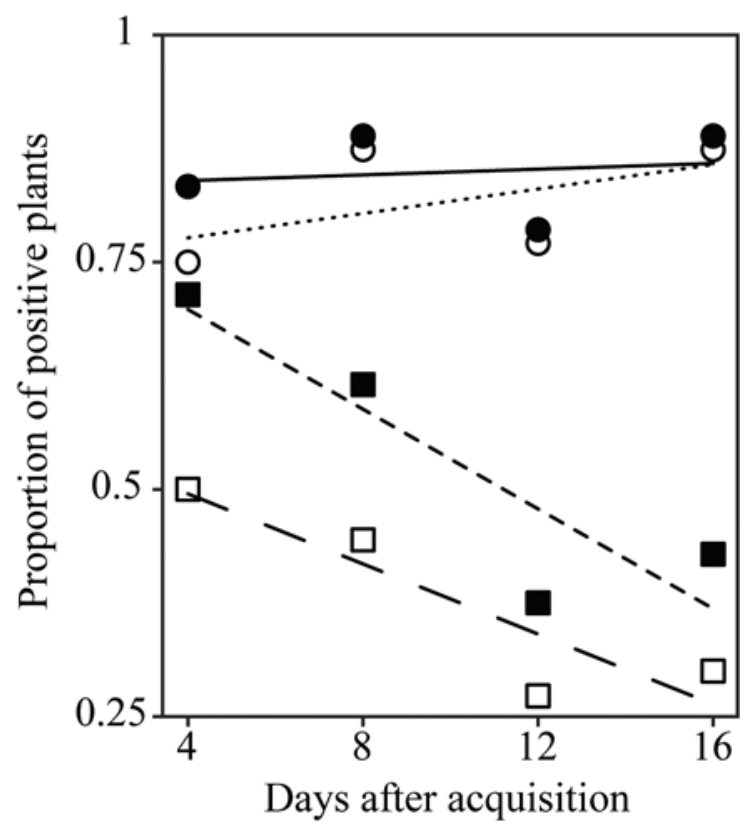

Fig. 7. Retention of Xylella fastidiosa wild-type and $r p f B$ mutant strains in leafhopper vectors as indicated by their frequency of transmission to grape plants in successive transfers. Individual leafhoppers were transferred to new grapevines every 4 days after acquiring the bacteria on source vines infected with either the $r p f B$ mutant (squares) or the wild type (circles). Solid symbols include the proportion of positive transmission events of a given Xylella fastidiosa strain for only those insects that transmitted the pathogen to at least one plant in the four-transfer series. Open symbols represent the proportion of all plants to which leafhoppers were given access that became infected at a given time after initial acquisition of the pathogen by feeding on infected plants (i.e., includes individuals that never transmitted the pathogen). Linear regressions: wild type/transmitting insects only (solid line, $r^{2}=0.027$ ), wild type/all insects (points, $r^{2}=0.321$ ), $r p f B$ mutant/transmitting insects only (small dashes, $\left.r^{2}=0.798\right)$ and, $r p f B$ mutant/all insects (long dashes, $r^{2}=0.817$ ). Some data points (wild type/all insects) were slightly moved for better visualization. deficient $r p f B$ mutant complemented with plasmids harboring either Xylella fastidiosa or Xanthomonas campestris rpfB did not have its DSF synthesis activity restored but that restoration could be conferred by addition of RpfF. In both Xanthomonas campestris and Xylella fastidiosa, RpfF appears to be absolutely required for DSF production (Barber et al. 1997; Newman et al. 2004). RpfB has been suggested to modify DSF downstream of RpfF activity (Barber et al. 1997). The results of this study are not inconsistent with this conjecture, because the fatty acids detected in an $r p f B$ mutant appear to be a subset of those found in the wild type. It seems likely that the Xanthomonas campestris eng:gfp-based DSF biosensor strain detects the effects of signal transduction mediated by the common fatty acid produced by both the $r p f B$ and wild-type strains of Xanthomonas campestris and Xylella fastidiosa. It is plausible that other downstream genes may respond differently to the different DSF species produced by the wild-type strain of either Xanthomonas campestris or Xylella fastidiosa. It has been shown that the eng-based reporter gene in Xanthomonas campestris was less responsive to CDSF than to either DSF or BDSF (He et al. 2010). Direct support for this conjecture was provided in this current study of Xylella fastidiosa. Although the expression of several virulence genes is strongly altered in an $r p f F$ mutant, only a subset of these genes was altered in an $r p f B$ mutant compared with the wild-type strain. This suggests that more than one somewhat overlapping signal transduction pathway may link various DSF species to expression of particular downstream genes. That is, RpfB-mediated modifications to the signaling molecule may be sensed by more than one receptor in this system. Newman and associates (2008) showed that DSF can be modified by a Pseudomonas strain in a way that alters its migration on TLC plates but the altered DSF was still detectable using the Xanthomonas campestris 8523 eng:gfp DSF biosensor used here. Likewise Xanthomonas campestris was shown to respond to a variety of related unsaturated fatty acids, although with different efficiencies (Wang et al. 2004). Thus, it seems likely that more than one form of DSF can serve as a signaling molecule in Xanthomonas campestris. Although there is no other direct evidence for such a parallel DSF-dependent cell-to-cell signaling regulon in Xanthomonas campestris, it has been suggested for Xylella fastidiosa (Chatterjee et al. 2008b). We suggest that a more detailed characterization of rpfB in Xanthomonas campestris is warranted to clarify its role in DSF synthesis. Despite the considerable efforts made to describe the DSF signal transduction pathways in both Xanthomonas campestris and Xylella fastidiosa, little is known of the mechanisms of DSF synthesis. The distal location of $r p f B$ in Xylella fastidiosa, away from $r p f F$, may enable more complex regulation of $\mathrm{RpfB}$ relative to $\mathrm{RpfF}$ than in Xanthomonas campestris, where their expression is apparently tightly linked. This may enable the production of varying amounts of different forms of DSF, which might contribute to its ability to coordinate its behavior in the more diverse habitats that it colonizes compared with Xanthomonas campestris. Accumulation of each of the three DSF molecules in Xanthomonas oryzae pv. oryzae was shown to be media dependent (He et al. 2010), suggesting that environmental context may be a factor mediating the relative proportion of DSF forms in different environments. Thus, such a phenomenon might be involved in differential DSF production by Xylella fastidiosa in insects and plants.

Although the role of RpfB in Xanthomonas campestris is not clear, its function in Xylella fastidiosa can be more easily studied in isolation of other $r p f$ signaling components because it is located elsewhere in the chromosome, away from the rpf operon. It appears that RpfB contributes more to insect colonization than to plant virulence. Xylella fastidiosa colonization 
of plants was not affected in the $r p f B$ mutant, as determined by symptom development in grapevines. RpfF and RpfC mutants of Xylella fastidiosa that are deficient in DSF synthesis and sensing, respectively, had dramatically increased and decreased virulence to grape compared with the wild-type strain (Chatterjee et all, 2008b; Newman et al. 2004), demonstrating that DSF strongly controls plant colonization in this pathogen. In contrast, the $\operatorname{rpf} B$ mutant appears to have virulence similar to that of the wild-type strain. The fact that $r p f B$ mutant insectinoculated plants took longer than the wild type to show symptoms is likely to be more a consequence of the number of cells inoculated by leafhoppers into plants than the inherent capacity of the strain to multiply and move within grapevines. Because colonization of vectors was affected in the $r p f B$ knockout, fewer cells may have been inoculated into plants, delaying development of large population sizes needed for symptom development and perhaps also reducing the number of inoculation sites, increasing the need for the pathogen to move more extensively between vessels. Experiments testing a similar hypothesis revealed that disease severity was higher and the time between inoculation and symptom development was shorter with increasing vector-feeding periods (Daugherty et al. 2009). Observed differences in symptom development after the onset of disease, with $r p f B$-infected plants apparently having a more rapid development of symptoms with time than the wild-type strain, may be a consequence of changing greenhouse environmental conditions during the course of the experiment. This observation needs to be further explored in more detail.

Although the contribution of RpfB to plant virulence was modest, a clear phenotype was found for its role in the colonization of leafhopper vectors and transmission to plants. Insects acquired the mutant from plants at slightly slower rates than the wild type, suggesting that initial acquisition of bacteria from sources was not very affected. This result also indicates that both $r p f B$ and the wild-type strains grow to comparable populations in the plant host, because the insects acquire the wild-type strain and the $\operatorname{rpf} B$ mutant to similar extents. However, retention of the $r p f B$ mutant in vectors was reduced, as shown by both direct detection methods (culturing) and transmission assays. Transmission studies are particularly useful assays because they represent the outcome of a complex pathogen-vector interaction, as measured by the infection of a new susceptible host. In these tests, the frequency of transmission of the wild-type strain of Xylella fastidiosa did not decrease with time after acquisition by insects that fed on source plants colonized by the wild-type strain but decreased linearly for the $r p f B$ mutant $\left(r^{2} \geq 0.8\right)$. Furthermore, initial acquisition rates were similar for both treatments, suggesting little if any effect of this gene on processes involved in early stages of vector colonization. It is possible that the family of fatty acids influenced by RpfB contributes to the late stage of vector colonization.

Xylella fastidiosa colonization of the foregut of xylem sapsucking leafhoppers is a complex process. Cells attach to the outermost cuticular layer of vectors, in a small region of the foregut that is constantly washed by dilute xylem sap being ingested in high volume (Almeida et al. 2005). Initially adhered cells are found attached parallel to that surface, whereas mature biofilms are composed of polarly attached cells perpendicular to the surface of the insect (Almeida and Purcell 2006). Although wild-type cells were found to form biofilms as previously shown on vectors (Almeida and Purcell 2006; Newman et al. 2004), a complex fimbriae-like network was observed on insects that fed on the $\operatorname{rpfB}$ mutant. We did not observe cells in any of these individuals, possibly because they were obscured by the amorphous material, although vectors were obviously colonized because Xylella fastidiosa was detected by culturing and was transmitted to plants. We speculate that the matrix represents an altered pattern of exopolymer production in the $r p f B$ mutant that may have partially blocked release of cells from the vector. Support for this conjecture is the finding that gumJ was highly overexpressed in the $r p f B$ mutant compared with the wild-type strain. Thus, we interpret these observations as evidence that early attachment of the $r p f B$ mutant to vectors is not affected but biofilm maturation is impacted, resulting in loss of attached cells over time.

The extensive differences in phenotype within vectors observed in the mutant suggest that $\mathrm{RpfB}$ is a component of a regulatory network involved in habitat-specific gene regulation. The DSF-sensing RpfC may preferentially recognize certain forms of DSF and the altered expression of RpfB may modulate the proportion of the various chemical forms of DSF that are produced. Although this hypothesis does not conflict with results obtained in our work and by other groups, it does imply that a parallel signaling system based on a family of DSF-like molecules exists in Xylella fastidiosa and, probably, Xanthomonas campestris, for which there is no bioinformatic evidence. This has been hypothesized for Xylella fastidiosa (Chatterjee et al. 2008b) and fits with our observations. In this scenario, the forms of DSF that would mediate Xylella fastidiosa plant colonization and initial adhesion to vectors might differ from those involved in colonization of insect vectors. Studies to test this conjecture would benefit from a better understanding of the chemical identity of the fatty acids involved in cell-to-cell signaling in Xylella fastidiosa.

\section{MATERIALS AND METHODS}

\section{Bacterial cultures.}

Xylella fastidiosa Temecula (Van Sluys et al. 2003) was used for all experiments. Xylella fastidiosa cultures were incubated at $28^{\circ} \mathrm{C}$; shaking at $160 \mathrm{rpm}$ was provided for liquid cultures. Liquid and solid media for Xylella fastidiosa were PW and PWG, respectively (Hill and Purcell 1995) with natamycin at 21.6 $\mu \mathrm{g} / \mathrm{ml}$ (Gist-brocades, The Netherlands). Xylella fastidiosa selective media contained kanamycin at $30 \mu \mathrm{g} / \mathrm{ml}$. Xanthomonas campestris strains 8004 (wild type) and 8550 (rpfB mutant) (Tang et al. 1991) were grown on King's B medium (KB) at $28^{\circ} \mathrm{C}$. When indicated, we added rifampicin at $100 \mu \mathrm{g} / \mathrm{ml}$, kanamycin at $50 \mu \mathrm{g} / \mathrm{ml}$, tetracycline at $10 \mu \mathrm{g} / \mathrm{ml}$, spectinomycin at $100 \mu \mathrm{g} / \mathrm{ml}$, and streptomycin at $50 \mu \mathrm{g} / \mathrm{ml}$ to media. The strains and plasmids used in this study are summarized in Table 2.

\section{Xylella fastidiosa rpfB knock-out strain KLN57.}

The $r p f B$ locus of Xylella fastidiosa was disrupted by allelic exchange after electroporation with pKLN57 (Fig. 1A), which was constructed as follows. A 2-kb region of the Xylella fastidiosa genome including most of the $r p f B$ coding sequence was amplified using primers $r p f B K O F X h o I$ and $r p f B K O R$ (Table 3), digested with XhoI and SalI, and ligated into the SalI site of pKLN2 (Newman et al. 2003) to make pKLN16. The Tn903 kanamycin resistance gene was amplified from pKLN56 (Newman et al. 2003) with primers Kan-2FRISma and Kan2RMCS, digested with BamHI and SmaI, and ligated into the BamHI and SmaI sites of pKLN16 to make pKLN57. pKLN57 was electroporated into Xylella fastidiosa and transformants selected as previously described (Newman et al. 2003). The disruption of the $r p f B$ locus was confirmed by PCR and Southern blotting. All amplifications were carried out using $P f u$ polymerase (Stratagene, La Jolla, CA, U.S.A.). We confirmed the insert sequences of all plasmids by sequencing.

\section{Complementation of the Xylella fastidiosa rpfB knockout.}

The $r p f B$ gene and its 400-bp upstream segment (presumably harboring its promoter) were amplified from the Xylella 
fastidiosa genome using the primers rpfBCOMP-F (TTGTAG TACTCGAGACGCTTAAAGATCCTAAAAAATTTCC) and rpfBCOMP-R (TTGTAGTACTCGAGAACTACTTGGGCTG AGCTGTGAATG). The PCR product was cloned into and the chromosomal based complementation vector $\mathrm{pAX} 1 \mathrm{Em}^{\mathrm{R}}$ (Matsumoto et al. 2009) through the XhoI restriction site presents on both primers and on the vector to generate $\mathrm{pAX} 1 \mathrm{Em}^{\mathrm{R}}$ $\operatorname{rpfB}$, and the new vector was transformed to Xylella fastidiosa rpfB mutant (KLN57) by exploiting its natural competence, as described by Kung and Almeida (2011). Recombinant cells were selected on PWG plates supplemented with kanamycin at $50 \mu \mathrm{g} / \mathrm{ml}$ and erythromycin at $50 \mu \mathrm{g} / \mathrm{ml}$. Integration of the $r p f B$ allele into NS1 was verified using primers NS1-F and NS1-R (Matsumoto et al. 2009). This vector provides constitutive expression of the cloned genes in hosts such as Xanthomonas spp. due to their lack of the lacI-encoded repressor.

\section{Xanthomonas campestris rpfB rescue.}

Rescue plasmids pKLN69 and pKLN70 (Newman et al. 2004) and pKLN71 and pKLN72 harbor the lac promoter fused to the $r p f F$ or $r p f B$ genes of Xanthomonas campestris (8004) or Xylella fastidiosa (Temecula). These plasmids were transferred into Xanthomonas campestris 8550 (rpfB mutant) (Tang et al. 1991) by conjugation. The $r p f B$ genes were amplified from the Xylella fastidiosa and Xanthomonas campestris genomes using primers XfrpfBFXhoI and XfrpfBRSpeI, and Xanthomonas campestrisrpfBFXhoI and Xanthomonas campestrisrpfBRSpeI, respectively (Table 2), and ligated into pGEM-T (Promega Corp., Madison, WI, U.S.A.) to make $\mathrm{p} X f r p f B-\mathrm{T}$ and $\mathrm{p} X c c r p f B-\mathrm{T}$, respectively. The $\operatorname{rpf} B$ genes were cut from these plasmids with XhoI and SpeI and ligated into the XhoI and SpeI sites of pBBR1MCS-3 (Kovach et al. 1995) to make pKLN71 and pKLN72. All amplifications were carried out using $P f u$ polymerase (Stratagene). The insert sequences of all plasmids were confirmed by sequencing.

\section{Expression analysis}

\section{by quantitative reverse-transcriptase-PCR.}

Quantification of transcript abundance was performed as previously published (Chatterjee et al. 2008b; Killiny and Almeida 2009a and b). Transcript abundance was determined as the mean of three independent biological replicates, each of which was repeated three times.

\section{Extraction and detection of DSF with reporter strain.}

For Xylella fastidiosa samples, stationary-phase PW liquid cultures (approximately 10 days), or PWG plate-grown cells (7 days), DSF was extracted twice with a one-third volume of water-extracted ethyl acetate similar to that of Chatterjee and associates (2008b). Supernatants were evaporated to completion under vacuum at $37^{\circ} \mathrm{C}$ and resuspended in methanol, pipetted onto sterile paper disks, allowed to dry, then pressed onto KB plates. Colonies of the Xanthomonas campestris DSF reporter strain 8523 (pKLN55) (Newman et al. 2004) were harvested from selective KB plates, resuspended to a concentration of approximately $5 \times 10^{8} \mathrm{CFU} / \mathrm{ml}$ in $10 \mathrm{mM}$ potassium phosphate buffer, and plated as a stripe of $20 \mu \mathrm{l}$ pipetted perpendicular to the paper disk. For Xanthomonas campestris samples, colonies of the 8523 (pKLN55) were harvested from selective $\mathrm{KB}$ plates, resuspended to a concentration of approximately $5 \times 10^{8} \mathrm{CFU} / \mathrm{ml}$ in $\mathrm{KB}$, and sprayed onto plates with an artist's airbrush. Plates were incubated at $28^{\circ} \mathrm{C}$ for $48 \mathrm{~h}$ before

Table 2. Bacterial strains and plasmids used in this study

\begin{tabular}{|c|c|c|}
\hline Strains & Description $^{a}$ & Source or reference \\
\hline \multicolumn{3}{|c|}{ Xanthomonas campestris pv. campestris } \\
\hline 8004 & Wild type $\left(\right.$ Rif $\left.^{r}\right)$ & Turner et al. 1984 \\
\hline 8550 & $r p f B:: \operatorname{Tn} 5\left(\mathrm{Rif}^{\mathrm{r}}, \mathrm{Km}^{\mathrm{r}}\right)$ & Tang et al. 1991 \\
\hline 8523 & $r p f F:: \operatorname{Tn} 5 \mathrm{lac}\left(\mathrm{Rif}^{\mathrm{r}}, \mathrm{Km}^{\mathrm{r}}\right)$ & Tang et al. 1991 \\
\hline \multicolumn{3}{|l|}{ Xylella fastidiosa } \\
\hline Temecula & Wild type & van Sluys et al. 2003 \\
\hline Temecula KLN57 & $r p f B, \mathrm{Km}^{\mathrm{r}}$ & This study \\
\hline Temecula KLN61 & $r p f F, \mathrm{Km}^{\mathrm{r}}$ & Newman et al. 2004 \\
\hline \multicolumn{3}{|l|}{ Plasmids } \\
\hline pKLN 55 & engXCA promoter-gfp, $\mathrm{Sp}^{\mathrm{r}}, \mathrm{St}^{\mathrm{r}}, \mathrm{mob}+$ & Newman et al. 2004 \\
\hline pGEM-5Zf $(+)$ & $\mathrm{Ap}^{\mathrm{r}}$ & Promega \\
\hline pXccrpfB-T & Xanthomonas campestris rpfB in pGEM-5Zf $(+), A^{r}$ & This study \\
\hline pXfrpfB-T & Xylella fastidiosa $r p f B$ in pGEM-5Zf $(+), A^{r}$ & This study \\
\hline pBBR1MCS-3 & pBBR1 ori, Tet ${ }^{\mathrm{r}}$ lacZ mob+ & Kovach 1995 \\
\hline pKLN69 & Xanthomonas campestris rpfF in pBBR1MCS-3 & Newman et al. 2004 \\
\hline pKLN70 & Xylella fastidiosa $r p f F$ in pBBR1MCS-3 & Newman et al. 2004 \\
\hline pKLN71 & Xanthomonas campestris rpfB in pBBR1MCS-3 & This study \\
\hline pKLN72 & Xylella fastidiosa rpfB in pBBR1MCS-3 & This study \\
\hline pKLN2 & Xylella fastidiosa oriC in pGEM-5Zf(+), Ap ${ }^{\mathrm{r}}$ & Newman et al. 2003 \\
\hline pKLN16 & Xylella fastidiosa $r p f B$ region in pGEM- $5 Z \mathrm{f}(+), \mathrm{Ap}^{\mathrm{r}}$ & This study \\
\hline pKLN57 & Xylella fastidiosa rpfB allelic exchange suicide plasmid, $X$. fastidiosa oriC, $\mathrm{Ap}^{\mathrm{r}}, \mathrm{Km}^{\mathrm{r}}$ & This study \\
\hline
\end{tabular}

${ }^{\mathrm{a}} \mathrm{Rif}^{\mathrm{r}}, \mathrm{Km}^{\mathrm{r}}, \mathrm{Sp}^{\mathrm{r}}, \mathrm{St}^{\mathrm{r}}$, and $\mathrm{Tet}^{\mathrm{r}}$ indicate resistant to rifampicin, kanamycin, spectinomycin, streptomycin, and tetracycline, respectively.

Table 3. List of synthetic oligonucleotides used in this study

\begin{tabular}{ll}
\hline Name & \multicolumn{1}{c}{ DNA sequence $\left(\mathbf{5}^{\prime}\right.$ to $\left.\mathbf{3}^{\prime}\right)$} \\
\hline rpfBKOFXhoI & TGTTTGCAATATCCAGTAGCGACCTT \\
$r p f B K O R$ & TGCAGGGTAATCTTAAAGTGCGTCGTA \\
Kan-2FRISma & GGAATTCCCGGGCTGTCTCTTATACACATCTCAACCA \\
Kan-2RMCS & ATATCAATTGAAGCTTGCATGCCTGCAGGTCGACTCTAGAGGATCCAACCAATTCTGATTAGAAAAA \\
& CTCA \\
Xanthomonas campestris rpfBFXhoI & ACTCGAGATAAGGAGGAAAAACATATGAGTCAGGCACGTCCTTGG \\
Xanthomonascampestris rpfBRSpeI & GACTAGTCTATGCCTTGGCCGCATCCCGCAATTCGCGA \\
XfrpfBFXhoI & ACTCGAGATAAGGAGGAAAAACATATGAGTCAAACACGTCCTTGG \\
XfrpfBRSpeI & CTACTTGGGCTGAGCTGTGAATGTGTTTGAT \\
\hline
\end{tabular}


viewing on a Zeiss SV11 stereoscope with Karmer epifluorescence/Optronix Color DEI450.

\section{DSF extraction and TLC fractionation.}

The DSF was extracted from Xylella fastidiosa and Xanthomonas campestris strains according to $\mathrm{He}$ and associates (2010) with slight modifications. Briefly, liquid cultures (KB for Xanthomonas campestris and PW for Xylella fastidiosa) were filtrated through $0.22-\mu \mathrm{m}$ filters and the $\mathrm{pH}$ was adjusted to 4.0 using hydrochloric acid. The culture supernatants then were extracted by partitioning with two volumes of water-saturated ethyl acetate and recovered in methanol. To analyze DSF species, TLC was carried out on silica gel $\mathrm{F}_{254}$ plates $(0.25$ $\mathrm{mm}$ ) (Merck, Amsterdam, The Netherlands) using the solvent system hexane/diethylether/acetic acid (80:20:1). To visualize the spots, plates were transferred after drying to a closed container, iodine crystals were added, and plates visualized after a period of 1 to 7 days.

\section{Pathogenicity assays.}

All experiments were performed on greenhouse-grown Vitis vinifera 'Cabernet Sauvignon' rooted cuttings or seedlings. During winter months, supplemental light was provided. For mechanical inoculation of grapevine cuttings, the droplet puncture method was employed (Hill and Purcell 1995). Grapevines were scored for the development of Pierce's disease symptoms at weekly intervals, and Xylella fastidiosa infection was verified by culturing bacteria from petiole macerates following Hill and Purcell (1995) followed by genotyping of bacterial colonies by PCR with primers $r p f B K O R$ " and "rpfBKOFXhoI. Symptoms were scored as none $=0$, slight scorching $=1$, moderate scorching $=2$, or severe scorching $=3$.

\section{Transmission tests.}

To determine whether the $r p f B$ mutant was vector transmitted to grapevines, we compared the transmission rates of the wild type and $r p f B$ mutant to grapevines by Graphocephala atropunctata (Signoret) (blue-green sharpshooter; Hemiptera, Cicadellidae), following general protocols previously described (Almeida and Purcell 2003). We initially conducted five tests that used the same experimental design. Individual uninfected adult leafhoppers were caged on symptomatic grapevines infected with KLN57 or the wild-type strain for a 4-day acquisition access period, then transferred to a healthy grapevine seedling for a 7-day inoculation access period. Grapevines were scored for disease symptoms as described above. Analysis of variance (ANOVA) was used to compare symptom development in plants inoculated with the wild-type and $r p f B$ mutant strains; ANOVA was carried out for each data point (days after inoculation, $\alpha=0.01$ ).

In one of these tests, we cultured Xylella fastidiosa from a subset of insects immediately after the acquisition and inoculation access periods to determine bacterial colonization patterns over time. Bacteria were cultured from insect heads as previously described (Almeida and Purcell 2003). In another replicate, leafhoppers that transmitted either strain to plants were processed for scanning electron miscroscopy observations of cell-colonization patterns. Thus, these individuals had combined 4-day acquisition and 7-day inoculation access periods. Xylella fastidiosa attachment sites in the foregut of vectors were analyzed by microscopy as previously done (Almeida and Purcell 2006; Newman et al. 2004).

In a second study, we determined the retention of the wild type and $r p f B$ mutants in vectors over time by transferring infective leafhoppers to new, uninfected grape seedling every 4 days for four successive 4-day inoculation access periods. We confined insects on infected plants for a 4-day acquisition access period. In this case, we measured transmission rates over time as a proxy for retention of the pathogen in the mouthparts of leafhoppers. We hypothesized, based on our culturing assays of leafhopper heads over time, that cells were being washed away from the mouthparts, which would reduce transmission rates with successive transfer of vectors to new plants. Consequently, retention is defined here as the capacity of vectors to transmit the pathogen to plants over time.

\section{ACKNOWLEDGMENTS}

We thank M. Dow for materials, including Xanthomonas campestris strains 8004 and 8550, and advice and discussion; E. Norberg and D. Hoenisch for providing assistance with grapevines; T. Wistrom, S. Rashby, and R. Koutsoukis for providing technical assistance with experiments; and S. Purcell for discussion and assistance. This material is based upon work supported by the National Science Foundation under a grant awarded to K. L. Newman in 2002 and by funding from the American Vineyard Foundation, California Competitive Grant Program for Research in Viticulture and Enology, and the Pierce's disease and glassy-winged sharpshooter control program of the California Department of Food and Agriculture and the United States Department of Agriculture.

\section{LITERATURE CITED}

Almeida, R. P. P., and Purcell, A. H. 2003. Transmission of Xylella fastidiosa to grapevines by Homalodisca coagulata (Hemiptera, Cicadellidae). J. Econ. Entomol. 96:264-271.

Almeida, R. P. P., and Purcell, A. H. 2006. Patterns of Xylella fastidiosa colonization on the precibarium of sharpshooter vectors relative to transmission to plants. Ann. Entomol. Soc. Am. 99:884-890.

Almeida, R. P. P., Blua, M. J., Lopes, J. R. S., and Purcell, A. H. 2005 Vector transmission of Xylella fastidiosa: Applying fundamental knowledge to generate disease management strategies. Ann. Entomol. Soc. Am. 98:775-786.

Barber, C. E., Tang, J. L., Feng, J. X., Pan, M. Q., Wilson, T. J. G., Slater, H., Dow, J. M., Williams, P., and Daniels, M. J. 1997. A novel regulatory system required for pathogenicity of Xanthomonas campestris is mediated by a small diffusible signal molecule. Mol. Microbiol. 24:555-566.

Camilli, A., and Bassler, B. L. 2006. Bacterial small molecule signaling pathways. Science 311:1113-1116.

Chatterjee, S., Almeida, R. P. P., and Lindow, S. E. 2008a. Living in two worlds: The plant and insect lifestyles of Xylella fastidiosa. Annu. Rev. Phytopathol. 46:243-271.

Chatterjee, S., Wistrom, C., and Lindow, S. E. 2008b. A cell-cell signaling sensor is required for virulence and insect transmission of Xylella fastidiosa. Proc. Natl. Acad. Sci. U.S.A. 105:2670-2675.

Chatterjee, S., Killiny, N., Almeida, R. P. P., and Lindow, S. E. 2010. Role of cyclic diGMP in Xylella fastidiosa biofilm formation, plant virulence and insect transmission. Mol. Plant-Microbe Interact. 23:1356-1363.

Cheng, Z., He, Y. W., Lim, S. C., Qamra, R., Walsh, M. A., Zhang, L. H., and Song, H. 2010. Structural basis of the sensor-synthase interaction in autoinduction of the quorum sensing signal DSF biosynthesis. Structure 18:1199-1209.

Daugherty, M. P., Bosco, D., and Almeida, R. P. P. 2009. Temperature mediates vector transmission efficiency: Inoculum supply and plant infection dynamics. Ann. Appl. Biol. 155:361-369.

Deng, Y., Wu, J., Tao. F., and Zhang, L.-H. 2011. Listening to a new language: DSF-based quorum sensing in gram negative bacteria. Chem. Rev. 111:160-173.

Dow, M. 2008. Diversification of the function of cell-cell signaling in regulation of virulence within plant pathogenic xanthomonads. Sci. Signal. 1 pe23.

Dow, J. M., Crossman, L., Findlay, K., He, Y. Q., Feng, J. X., and Tang, J. L. 2003. Biofilm dispersal in Xanthomonas campestris is controlled by cell-cell signaling and is required for full virulence to plants. Proc. Natl. Acad. Sci. U.S.A. 100:10995-11000.

Guilhabert, M. R., and Kirkpatrick, B. C. 2005. Identification of Xylella fastidiosa antivirulence genes: Hemagglutinin adhesins contribute to $X$. fastidiosa biofilm maturation and colonization and attenuate virulence. Mol. Plant-Microbe Interact. 18:856-868.

He, Y.-W., and Zhang, L.-H. 2008. Quorum sensing and virulence regulation in Xanthomonas campestris. FEMS (Fed. Eur. Microbiol. Soc.) Mi crobiol. Rev. 32:842-857.

He, Y.-W., Wang, C., Zhou, L., Song, H., Dow, J. M., and Zhang, L.-H. 2006. Dual signaling functions of the hybrid sensor kinase RpfC of 
Xanthomonas campestris involved either phosphorelay or receiver domain-protein interaction. J. Biol. Chem. 281:33414-33421.

He, Y.-W., Wu, J., Cha, J. S., and Zhang, L.-H. 2010. Rice bacterial blight pathogen Xanthomonas oryzae produces multiple DSF-family signals in regulation of virulence factor production. BMC Microbiol. 10:187.

Hill, B. L., and Purcell, A. H. 1995. Multiplication and movement of Xylella fastidiosa within grapevine and four other plants. Phytopathology 85:1368-1372.

Killiny, N., and Almeida, R. P. P. 2009a. Host structural carbohydrate induces vector transmission of a bacterial plant pathogen. Proc. Natl. Acad. Sci. U.S.A. 106:22416-22420.

Killiny, N., and Almeida, R. P. P. 2009b. Xylella fastidiosa afimbrial adhesins mediate cell transmission to plants by leafhopper vectors. Appl. Environ. Microbiol. 75:521-528.

Kovach, M. E., Elzer, P. H., Hill, D. S., Robertson, G. T., Farris, M. A., Roop, R. M., and Peterson, K. M. 1995. Four new derivatives of broad host range cloning vector pBRR1MCS, carrying different antibiotic resistance cassettes. Gene 166:175-176.

Kung, S. H., and Almeida, R. P. P. 2011. Natural competence and recombination in the plant pathogen Xylella fastidiosa. Appl. Environ. Microbiol. 77:5278-5284.

Matsumoto, A., Young, G. M., and Igo, M. M. 2009. Chromosome-based genetic complementation system for Xylella fastidiosa. Appl. Environ. Microbiol. 75:1679-1687.

Newman, K. L., Almeida, R. P. P., Purcell, A. H., and Lindow, S. E. 2003. Use of a green fluorescent strain for analysis of Xylella fastidiosa colonization of Vitis vinifera. Appl. Environ. Microbiol. 69:7319-7327.

Newman, K. L., Almeida, R. P. P., Purcell, A. H., and Lindow, S. E. 2004 Cell-cell signaling controls Xylella fastidiosa interactions with both insects and plants. Proc. Natl. Acad. Sci. U.S.A. 101:1737-1742.

Newman, K. L., Chatterjee, S., Ho, K. A., and Lindow, S. E. 2008. Virulence of plant pathogenic bacteria attenuated by degradation of fatty acid cell- cell signaling factors. Mol. Plant-Microbe Interact. 21:326-334.

Pruzzo, C., Vezzulli, L., and Colwell, R. R. 2008. Global impact of Vibrio cholerae interactions with chitin. Environ. Microbiol. 10:1400-1410.

Tang, J. L., Liu, Y. N., Barber, C. E., Dow, J. M., Wootton, J. C., and Daniels, M. J. 1991. Genetic and molecular analysis of a cluster of rpf genes involved in positive regulation of synthesis of extracellular enzymes and polysaccharide in Xanthomonas campestris pathovar campestris. Mol. Gen. Genet. 226:409-417.

Van Sluys, M. A., de Oliveira, M. C., Monteiro-Vitorello, C. B., Miyaki, C. Y., Furlan, L. R., Camargo, L. E., da Silva, A. C., Moon, D. H., Takita, M. A., Lemos, E. G., Machado, M. A., Ferro, M. I., da Silva, F. R., Goldman, M. H., Goldman, G. H., Lemos, M. V., El-Dorry, H., Tsai, S. M., Carrer, H., Carraro, D. M., de Oliveira, R. C., Nunes, L. R., Siqueira, W. J., Coutinho, L. L., Kimura, E. T., Ferro, E. S., Harakava, R., Kuramae, E. E., Marino, C. L., Giglioti, E., Abreu, I. L., Alves, L. M. do Amaral, A. M., Baia, G. S., Blanco, S. R., Brito, M. S., Cannavan, F S., Celestino, A. V., da Cunha, A. F., Fenille, R. C., Ferro, J. A., Formighieri, E. F., Kishi, L. T., Leoni, S. G., Oliveira, A. R., Rosa, V. E. Jr., Sassaki, F. T., Sena, J. A., de Souza, A. A., Truffi, D., Tsukumo, F., Yanai, G. M., Zaros, L. G., Civerolo, E. L., Simpson, A. J., Almeida, N. F. Jr., Setubal, J. C., and Kitajima, J. P. 2003. Comparative analysis of the complete genome sequences of Pierce's disease and citrus variegated chlorosis strain of Xylella fastidiosa. J. Bacteriol. 185:1018-1026.

Wang, L. H., He, Y., Gao, Y., Wu, J. E., Dong, Y. H., He, C., Wang, S. X., Weng, L. X., Xu, J. L., Tay, L., Fang, R. X., and Zhang, L. H. 2004. A bacterial cell-cell communication signal with cross-kingdom structural analogues. Mol. Microbiol. 51:903-912.

Wilson, T. J., Bertrand, N., Tang, J. L., Feng, J. X., Pan, M. Q., Barber, C. E., Dow, J. M., and Daniels, M. J. 1998. The rpfA gene of Xanthomonas campestris pathovar campestris, which is involved in the regulation of pathogenicity factor production, encodes an aconitase. Mol. Microbiol. 28:961-970 